\title{
Safety of immediate use of nonsteroidal antiinflammatory drugs after pediatric craniotomy for tumor
}

\author{
Cody L. Nesvick, MD, Soliman Oushy, MD, David J. Daniels, MD, PhD, and Edward S. Ahn, MD \\ Department of Neurologic Surgery, Mayo Clinic, Rochester, Minnesota
}

OBJECTIVE Postoperative pain can limit the recovery of children undergoing craniotomy for tumor resection, and pain management is highly variable between institutions and practitioners. Nonsteroidal antiinflammatory drugs (NSAIDs) are effective in treating postoperative pain following craniotomy, but their use has been limited by concerns about postoperative hemorrhage. The risk of postoperative hemorrhage is not insignificant in patients undergoing craniotomy for tumor resection. No study has specifically addressed the safety of NSAIDs in the immediate postoperative setting following craniotomy for tumor resection in pediatric patients.

METHODS The authors performed a retrospective cohort study in patients younger than 18 years of age who underwent craniotomy for tumor resection at a single tertiary referral center between 2009 and 2019. The study outcomes were 1) postoperative hemorrhage requiring return to the operating room for decompression, evacuation, or CSF diversion for hemorrhage-associated hydrocephalus; and 2) more-than-minimal hemorrhage on routine postoperative imaging. Patients receiving any NSAID in the hospital formulary on the same day as surgery (postoperative day zero [POD0]) were designated as such.

RESULTS Two hundred seventy-six children underwent 308 craniotomies for tumor resection over the study period. One hundred fifty-four patients (50.0\%) received at least one dose of an NSAID on POD0. Six patients (1.9\%) required a return to the operating room for a hemorrhagic complication, including 3 who received an NSAID on POD0 (OR 1.00, $95 \% \mathrm{Cl} 0.20-5.03)$. Seventeen patients (6.3\% of patients imaged) had more-than-minimal hemorrhage on routine postoperative imaging, 9 of whom received an NSAID on POD0 (OR 1.08, 95\% Cl 0.40-2.89).

CONCLUSIONS Use of NSAIDs on PODO was not associated with either an increased risk of hemorrhage requiring a return to the operating room or asymptomatic hemorrhage on routine postoperative imaging. The overall incidence of clinically significant postoperative intracranial hemorrhage is low. These data support the use of NSAIDs as a safe measure for pain control in the postoperative setting for children undergoing craniotomy for tumor resection.

https://thejns.org/doi/abs/10.3171/2020.4.PEDS2055

KEYWORDS nonsteroidal antiinflammatory drug; NSAID; craniotomy; tumor; pain

$\mathrm{S}$ URGERY is a risk factor for persistent, long-term opiate use in both adults and children. The perioperative period is often the first time a child is exposed to narcotics, and approximately $5 \%$ of children undergoing surgery will continue to use narcotics more than 3 months after surgery. ${ }^{1,2}$ Despite an overall decline in the self-reported rates of opioid use in children, the rate of opioidrelated hospitalizations has continued to rise in this age group. ${ }^{3}$ Opioid dependence is an ongoing problem in the US pediatric population that demands the attention of the surgical community.
In the era of routine postoperative opioid use, there is increasing interest in developing efficacious, opioid-sparing postoperative pain-control regimens. Nonsteroidal antiinflammatory drugs (NSAIDs) are reversible cyclooxygenase (COX) inhibitors that exert analgesic effects centrally via reduction in CNS prostaglandin production. ${ }^{4}$ Prior studies have demonstrated that NSAIDs are efficacious and cost-effective in treating craniotomy-related pain in the immediate postoperative period. ${ }^{5-8}$ However, NSAIDs are often not used following craniotomy, due to the antiplatelet effects of COX inhibition. ${ }^{9-11}$ Although most re-

ABBREVIATIONS COX = cyclooxygenase; EVD = external ventricular drain; IQR = interquartile range; IVH = intraventricular hemorrhage; JPA = juvenile pilocytic astrocytoma; NSAID = nonsteroidal antiinflammatory drug; PODO = postoperative day zero; RCT = randomized controlled trial; SAH = subarachnoid hemorrhage; VPS = ventriculoperitoneal shunt.

SUBMITTED January 27, 2020. ACCEPTED April 6, 2020.

INCLUDE WHEN CITING Published online June 5, 2020; DOI: 10.3171/2020.4.PEDS2055. 
cent cohort studies have demonstrated no increased risk of hemorrhage-related complications following NSAID use in patients undergoing craniotomy, ${ }^{5,6,12,13}$ this is not consistent across the literature. ${ }^{14,15}$ To date, few studies have specifically assessed the safety of NSAID use in children undergoing craniotomy, ${ }^{16,17}$ and no study has specifically assessed NSAID use in the immediate postoperative period (i.e., postoperative day zero [POD0]), when the risk of postoperative hemorrhage is highest. ${ }^{18}$

Postoperative hemorrhage is a potential major complication following craniotomy, with approximately $1 \%-2 \%$ of patients requiring a return to the operating room for hematoma evacuation..$^{19,20}$ Due to abnormal tumor vasculature and challenging local vascular anatomy, tumor surgeons are often reluctant to administer any medication that may potentially increase the risk of postoperative hemorrhage. The aim of this study was to evaluate the risk of hemorrhage-related adverse outcomes in children undergoing craniotomy for tumor resection and receiving NSAIDs for pain control in the immediate postoperative period.

\section{Methods}

This study was approved by the institutional review board of Mayo Clinic, and consent was waived due to minimal risk to research subjects. We performed a retrospective cohort study to determine the relative risk of hemorrhage-related outcomes in pediatric patients receiving NSAIDs in the immediate postoperative period. The experimental cohort was defined as patients who had received an NSAID on POD0; control patients received no NSAIDs on POD0.

A prospectively collected database of patients undergoing neurological surgery at Mayo Clinic was mined for cases of pediatric patients undergoing tumor resection. This study included only patients younger than 18 years of age undergoing craniotomy for tumor resection, including bony tumors. Patients undergoing craniotomy for alternative indications (i.e., cortical resection and grid placement for epilepsy, arachnoid cyst fenestration, suboccipital decompression for Chiari malformation, and decompressive craniectomy for trauma) were excluded. Patients undergoing surgery for biopsy only were also excluded. We restricted the time frame of our study to January 2009-June 2019 to accurately reflect contemporary pathology, demographics, and clinical practice. Because this was a retrospective cohort study by design, the population under study was a sample of convenience, and sample size calculation for the purposes of statistical power estimation was deferred.

The medication administration records of all patients meeting inclusion and exclusion criteria were searched for instances of administration of any NSAID in the Mayo Clinic hospital formulary. This included aspirin, ketorolac, ibuprofen, diclofenac, etodolac, fenoprofen, flurbiprofen, indomethacin, ketroprofen, meclofenamate, nabumetone, naproxen, nepafenac, piroxicam, sulindac, and tolmetin. Patients receiving any of these medications on the same date as surgery were defined as receiving a POD0 NSAID. Medication and dose administration data were extracted for each patient. For reference, ketorolac was dosed 0.5 $\mathrm{mg} / \mathrm{kg} /$ dose every $6-8$ hours up to $15 \mathrm{mg}$ per dose. For older children and adolescents with no underlying renal pathology, a one-time dose of $30 \mathrm{mg}$ was allowed. Following postoperative stabilization, patients were transitioned to oral medication exclusively (i.e., ibuprofen) as soon as possible to minimize the risk of gastrointestinal and renal adverse effects.

The primary outcome for this study was return to the operating room for a hemorrhage-related complication including hematoma evacuation, decompression, external ventricular drain (EVD) or ventriculoperitoneal shunt (VPS) placement for hydrocephalus related to subarachnoid hemorrhage (SAH) or intraventricular hemorrhage (IVH). The secondary outcome for this study was the presence of more-than-minimal hemorrhage on routine postoperative imaging within 7 days of surgery. In line with the definition used by Richardson et al., ${ }^{17}$ we defined this outcome by the radiologist's interpretation of the degree of hemorrhage at the time of image acquisition. Description of hemorrhage or blood products as "minimal," "mild," "thin," "small," or "expected" were not considered positive findings for this outcome. All instances of morethan-minimal hemorrhage for the craniotomy performed were confirmed independently by two observers (C.L.N., E.S.A.) who were blinded to NSAID administration status.

Descriptive statistics are presented as median values with interquartile ranges (IQRs). Statistical comparisons were made using odds ratios and $95 \%$ confidence intervals where indicated. Statistical analyses were performed using JMP 14.1 (SAS Institute).

\section{Results}

Two hundred seventy-six patients underwent 308 craniotomies for tumor resection over the specified time period. Demographic data for the patients included in this study are presented in Table 1. At least one dose of an NSAID was administered on the same date as surgery in exactly half of patients, with ketorolac being the sole NSAID administered in all but 3 patients. The spectrum of tumor pathology in patients included in this study is shown in Table 2. Craniotomies were performed most commonly for focal astrocytic tumors such as juvenile pilocytic astrocytoma (JPA; 27.6\%), embryonal tumors (14.6\%), and infiltrating glial tumors including glioblastoma and diffuse astrocytoma (13.6\%).

Primary and secondary outcome data are presented in Table 3. Six patients (1.9\%) required a return to the operating room for hemorrhage-associated complications, including 4 patients $(1.3 \%)$ who returned for decompression or hematoma evacuation and 2 patients $(0.6 \%)$ who required CSF diversion for SAH/IVH-associated hydrocephalus. Details of patients requiring a return to the operating room are shown in Table 4. Postoperative imaging was obtained within 7 days of surgery in 268 cases (87.0\%), including 253 (82.1\%) with MRI. Of the 40 patients who did not receive routine postoperative imaging, $30(75 \%)$ underwent craniotomy for resection of extradural or intradural, extraaxial tumors. More-than-minimal hemorrhage was observed in 17 cases (6.3\%). Illustrative cases are shown in Figs. 1 and 2. 
TABLE 1. Characteristics of 276 pediatric patients who underwent craniotomies for tumor resection

\begin{tabular}{lc}
\hline \multicolumn{1}{c}{ Factor } & No. $(\%)$ \\
\hline Patients & 276 \\
\hline Craniotomies for tumor resection & 308 \\
\hline Median age in yrs, IQR & $11,5-15$ \\
\hline Female sex & $144(46.8 \%)$ \\
\hline Received any PODO NSAIDs & $154(50.0 \%)$ \\
\hline Ketorolac & $151(49.0 \%)$ \\
\hline Median total same-day dose in mg, IQR & $20,15-30$ \\
\hline Ibuprofen & $1(0.3 \%)$ \\
\hline Total same-day dose in mg & 300 \\
\hline Ketorolac \& ibuprofen & $2(0.6 \%)$ \\
\hline Median total same-day doses in mg & 22 ketorolac; 500 ibu- \\
& profen \\
\hline
\end{tabular}

Outcome comparisons are presented in Table 5. There was no significant difference in the rate of return to the operating room between patients who received or did not receive POD0 NSAIDs (OR 1.00, 95\% CI 0.20-5.03). There remained no difference between groups when patients requiring a delayed return to the operating room for CSF diversion were excluded from the analysis (OR 3.04, 95\% CI 0.31-29.5). There was also no significant difference in the rate of more-than-minimal hemorrhage on postoperative imaging between groups (OR 1.08, 95\% CI 0.40-2.89). In a nested case-control analysis that included only patients who received NSAIDs, the dose of ketorolac was not significantly different in patients who had morethan-minimal hemorrhage on postoperative imaging $(\mathrm{p}=$ $0.94)$. Because only 3 patients who received NSAIDs required a return to the operating room, statistical comparisons to this group were not performed, but ketorolac doses were not qualitatively different between patients requiring a return to the operating room (range 15-30 mg POD0 ketorolac) and those who did not (median $20 \mathrm{mg}$, IQR 15-30 mg POD0 ketorolac).

\section{Discussion}

It is widely believed that administration of NSAIDs in the perioperative period is unsafe due to an increased risk of postoperative hemorrhage. The growing need for nonnarcotic-based postoperative pain regimens has rekindled interest in using NSAIDs for postoperative pain control. Ketorolac and ibuprofen are the most commonly used NSAIDs in postsurgical patients, and ketorolac specifically has been shown to inhibit platelet function in vivo and prolong the bleeding time of healthy volunteers. ${ }^{9-11}$

Multiple studies have assessed the efficacy and safety of NSAID administration in adults undergoing elective craniotomy. Two case-control studies demonstrated that patients with symptomatic hemorrhage requiring a return to the operating room were more likely to have received NSAIDs around the time of surgery. In a case-control study of 6668 patients undergoing elective craniotomy in the United Kingdom, Palmer and colleagues found that $43 \%$ of patients undergoing intracranial or neck surgery
TABLE 2. Pathology of patients included in this study

\begin{tabular}{lr}
\hline \multicolumn{1}{c}{ Pathology* } & No. $(\%)$ \\
\hline Other astrocytic tumors; JPA, PXA, PMA & $85(27.6 \%)$ \\
\hline Embryonal tumors; medulloblastoma, ATRT & $45(14.6 \%)$ \\
\hline $\begin{array}{l}\text { Diffuse astrocytic \& oligodendroglial tumors; glioblas- } \\
\text { toma, DMG, diffuse astrocytoma }\end{array}$ & $42(13.6 \%)$ \\
\hline Neuronal \& mixed neuronal-glial tumors & $35(11.4 \%)$ \\
\hline Ependymoma & $18(5.8 \%)$ \\
\hline Craniopharyngioma & $11(3.6 \%)$ \\
\hline Schwannoma & $10(3.2 \%)$ \\
\hline Meningioma; grades I \& II & $9(2.9 \%)$ \\
\hline Cavernomas & $7(2.3 \%)$ \\
\hline Mesenchymal, non-meningothelial tumors; hemangio- & $7(2.3 \%)$ \\
$\quad$ blastoma, Ewing sarcoma & $7(2.3 \%)$ \\
\hline Choroid plexus tumors; CPP, CPC & $6(1.9 \%)$ \\
\hline Germ cell tumors & $3(1.0 \%)$ \\
\hline Paraganglioma & $3(1.0 \%)$ \\
\hline Tumors of pineal region; pineocytoma, pineoblastoma & $2(0.6 \%)$ \\
\hline Metastatic tumors & $1(0.3 \%)$ \\
\hline Histiocytosis X & $18(5.8 \%)$ \\
\hline Other &
\end{tabular}

ATRT = atypical teratoid rhabdoid tumor; $\mathrm{CPC}=$ choroid plexus carcinoma; $\mathrm{CPP}=$ choroid plexus papilloma; $\mathrm{DMG}=$ diffuse midline glioma; $\mathrm{PMA}=$ pilomyxoid astrocytoma; PXA = pleomorphic xanthoastrocytoma.

* Loosely defined by WHO 2016 classification of brain tumors.

who subsequently required hematoma evacuation had used NSAIDs or aspirin in the 2 weeks preceding surgery. ${ }^{15}$ However, no data were presented on how many patients took aspirin versus other NSAIDs, which NSAIDs were used, or whether any of these patients received an NSAID in the postoperative period. In another case-control study of 42,359 patients undergoing elective craniotomy in China, Jian et al. found that patients with postoperative intracranial hemorrhage requiring surgery were more likely to have received intraoperative flurbiprofen, a nonselective COX inhibitor. ${ }^{14}$ However, there was no association between postoperative flurbiprofen administration and likelihood of symptomatic hemorrhage.

There are 3 randomized controlled trials (RCTs) of NSAID efficacy following craniotomy in adults that contain data on safety, and 1 historical cohort study of NSAID use that assessed the risk of hemorrhage requiring evacuation. Magni et al. presented a historical cohort study of 4086 patients undergoing elective intracranial surgery, and found that those receiving ketorolac did not have a higher risk of postoperative hemorrhage requiring surgery. ${ }^{12}$ In a nested case-control analysis within this cohort, there was no association between ketorolac administration and need to return to the operating room for hematoma evacuation. Of note, however, timing to NSAID administration was not mentioned in this study. In the 3 RCTs of NSAID efficacy in craniotomy, no instances of postoperative hemorrhage have been reported, but the size of these studies is considerably smaller than in the other studies presented here (range 45-200 patients). ${ }^{5,6,13}$ 
TABLE 3. Outcomes in 268 patients who underwent craniotomies for tumor resection in whom postoperative imaging was available

\begin{tabular}{lc}
\hline \multicolumn{1}{c}{ Factor } & No. (\%) \\
\hline Any postop imaging & $268(87.0 \%)$ \\
\hline MRI & $253(82.1 \%)$ \\
\hline CT & $15(4.9 \%)$ \\
\hline More-than-minimal hemorrhage on imaging & $17(6.3 \%)$ \\
\hline Median total same-day dose in mg, range & $8,0-40$ \\
\hline Return to op room for any hemorrhage-related complication & $6(1.9 \%)$ \\
\hline Return to op room for clot evacuation or decompression & $4(1.3 \%)$ \\
\hline Delayed return to op room for EVD or VPS placement in setting of prior SAH/IVH & $2(0.6 \%)$ \\
\hline Median total same-day dose in mg, range & $7.5,0-30$ \\
\hline
\end{tabular}

* Within 7 days of surgery.

Three previous studies have specifically investigated the safety and efficacy of NSAIDs for treatment of pain following craniotomy in children. Bauer et al. presented a series of 51 children who underwent craniotomy for tumor biopsy or resection and received alternating, scheduled doses of ibuprofen and acetaminophen. ${ }^{16}$ Only 1 patient $(1.9 \%)$ had more-than-minimal hemorrhage after surgery, $9(17.6 \%)$ had a small amount of hemorrhage, and none required a return to the operating room. Forty-four patients (86.3\%) required no or minimal narcotics after surgery. Smyth et al. presented a historical cohort study of 50 children who underwent suboccipital decompression for Chiari malformation and were treated with either scheduled or as-needed ibuprofen and acetaminophen. ${ }^{21}$ No data regarding hemorrhage-related outcomes were presented, but patients receiving scheduled nonnarcotic medications had a shorted length of stay and lower narcotic requirements. In a study of 1451 cranial procedures in children, Richardson et al. found no association between ketorolac administration within 24 hours prior to or 72 hours following surgery and either need to return to the operating room for hematoma evacuation or presence of hemorrhage on postoperative imaging. ${ }^{17}$ The majority of patients in their study underwent craniotomy for cortical resection for epilepsy, suboccipital decompression for Chiari malformation, or
VPS placement. It is unclear how many patients in their study underwent tumor resection.

The study presented here is the largest investigation of NSAID safety in children undergoing craniotomy for tumor, and the first to specifically assess the safety of immediate use after surgery. In contrast to previous reports, routine postoperative imaging was obtained in the majority of patients in this study, so the rate of asymptomatic but volumetrically significant hemorrhage is therefore likely to be representative of the true rate following craniotomy for tumor resection. We comprehensively searched our hospital formulary for all possible NSAIDs used and applied a broad definition of postoperative hemorrhage to capture as many potential associations as possible. Importantly, there was a sufficient distribution of postoperative NSAID use (half of patients received a POD0 NSAID) that outcome comparisons were statistically possible.

However, the results of our study should be interpreted with several limitations in mind. Most notably, the sample size in the current study is smaller than some historical cohort and case-control studies of NSAID use following craniotomy for other indications. As a retrospective cohort study, our sample size was limited by convenience of the sample. If a prospective study were undertaken, the low incidence of the outcome of interest would require a

TABLE 4. Features of patients requiring a return to the operating room

\begin{tabular}{lclcc}
\hline \multicolumn{1}{c}{ Pathology } & Age & \multicolumn{1}{c}{ Exact Complication } & $\begin{array}{c}\text { Received POD0 } \\
\text { NSAIDs }\end{array}$ & $\begin{array}{c}\text { Extent of } \\
\text { Resection }\end{array}$ \\
\hline $\begin{array}{l}\text { Recurrent pineal region oligoden- } \\
\text { droglioma }\end{array}$ & $17 \mathrm{yrs}$ & Epidural hematoma & Yes & STR \\
\hline SEGA $\times 2$ & $8 \mathrm{yrs}$ & $\mathrm{IPH}$, venous infarction* & Yes & GTR \\
\hline Medulloblastoma & $8 \mathrm{yrs}$ & $\mathrm{Remote}^{*}$ epidural hematoma & No & GTR \\
\hline Hemispheric glioblastoma & $7 \mathrm{yrs}$ & $\mathrm{IPH}^{*}$ & Yes & STR \\
\hline DIG/DIA & $4 \mathrm{mos}$ & $\mathrm{SAH}$ & No & STR \\
\hline JPA & $10 \mathrm{yrs}$ & $\mathrm{SAH}, \mathrm{IVH}$ & No & STR \\
\hline
\end{tabular}

DIG/DIA = dysembryoplastic infantile ganglioglioma/dysembryoplastic infantile astrocytoma; GTR = gross-total resection; IPH = intraparenchymal hemorrhage; SEGA = subependymal giant cell astrocytoma; STR = subtotal resection.

* Decision to return to operating room for decompression also based on significant edema related to venous infarction or residual tumor-associated vasogenic edema. 

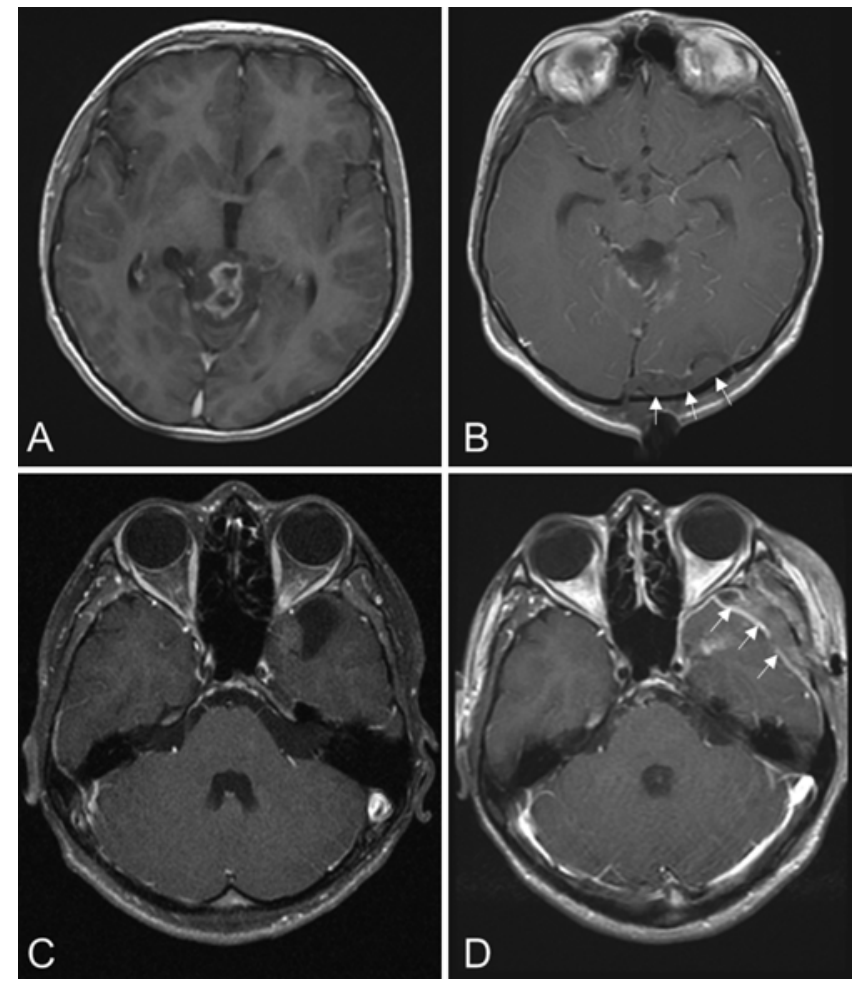

FIG. 1. Examples of symptomatic and asymptomatic hemorrhage encountered in this study. A and B: Axial postcontrast T1-weighted MR images in a 17-year-old boy with a recurrent pineal-region oligodendroglioma. The tumor was resected via a left-sided occipital transtentorial approach. The patient received a single $20-\mathrm{mg}$ dose of ketorolac for pain control on POD0. An occipital epidural hematoma was noted on postoperative MRI (white arrows), and the patient subsequently developed a homonymous hemianopia. His craniotomy was reopened, his hematoma evacuated, and his visual fields were grossly normal to confrontation at last clinical follow-up. C and D: Axial postcontrast T1-weighted MR images in a 14-year-old boy demonstrating a lightly enhancing nodule in the medial left anterior temporal lobe with an associated cyst. The patient underwent resection of the lesion via a left temporal craniotomy, and pathology was consistent with a JPA. He received a total of $40 \mathrm{mg}$ ketorolac on POD0. A left temporal epidural hematoma was discovered on postoperative MRI (white arrows). The patient remained asymptomatic, and the hemorrhage was observed.

very large sample size. For instance, using the incidence of a similar cohort study, ${ }^{17}$ assume the rate of returning to the operating room for hematoma evacuation following craniotomy is $0.5 \%$. With $80 \%$ power to detect a doubling in risk of this outcome following NSAID administration, 9346 patients would be required for the study. If the incidence rate is $2 \%$, as in this study, 2282 patients would be required, which is still a prohibitively large number. Although an RCT to definitively demonstrate safety of postoperative NSAID use in this patient population would be ideal, it would probably have to include children undergoing craniotomy for additional indications. Cohort and case-control studies, in particular, remain excellent tools to study events with low incidence and remain highly valuable in the absence of a large population under study. Similarly, the use of large administrative databases would overcome the issue of sample size, but as others have
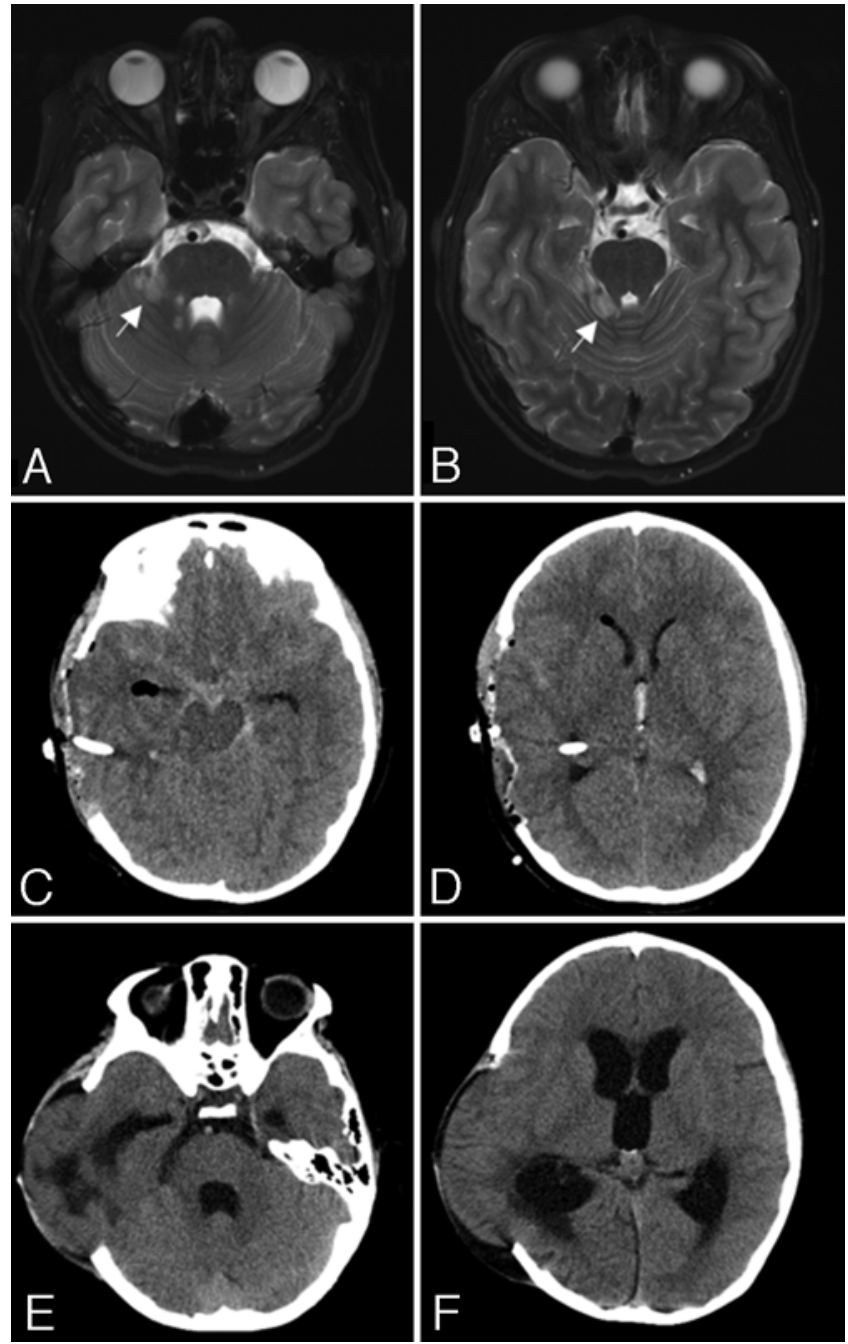

FIG. 2. Example of SAH and IVH requiring a return to the operating room for treatment of communicating hydrocephalus. A and B: Axial T2weighted MR images demonstrating a multifocal, T2-hyperintense lesion in the right cerebellar hemisphere in a 10-year-old boy (white arrows). No enhancement was noted following administration of gadolinium. The lesions were resected using a subtemporal transtentorial approach. Due to a significant amount of cerebral edema encountered at surgery, the bone flap was left off, and a right EVD was placed. C and D: Diffuse $\mathrm{SAH}$ as well as IVH affecting the lateral and third ventricles was noted on CT scan postoperatively. The patient received no NSAIDs on PODO. He was ultimately weaned from the EVD. E and F: Continued surveillance imaging demonstrated progressive ventriculomegaly in a pattern consistent with communicating hydrocephalus following EVD removal. The patient returned to the operating room for a second EVD placement, and he subsequently underwent VPS placement.

pointed out, ${ }^{22,23}$ these databases often rely on billing codes for diagnoses, and many specific outcomes (i.e., return to the operating room for a hemorrhage-related complication) are not routinely coded in the electronic medical record. A multiinstitutional retrospective cohort study may provide an excellent next step, providing more statistical power without losing granularity and accuracy of these data points.

Second, as a retrospective cohort study, this investi- 
TABLE 5. Outcome comparisons in pediatric patients who underwent craniotomies for tumor resection

\begin{tabular}{|c|c|c|c|}
\hline \multirow[b]{2}{*}{ Factor } & \multicolumn{2}{|c|}{$\begin{array}{l}\text { No. Receiving } \\
\text { PODO NSAIDs }\end{array}$} & \multirow[b]{2}{*}{ OR (95\% Cl) } \\
\hline & Yes & No & \\
\hline $\begin{array}{l}\text { More-than-minimal hemorrhage } \\
\text { on imaging }\end{array}$ & 9 & 8 & $1.08(0.40-2.89)$ \\
\hline $\begin{array}{l}\text { Return to op room for clot evacu- } \\
\text { ation or decompression }\end{array}$ & 3 & 1 & $3.04(0.31-29.5)$ \\
\hline $\begin{array}{l}\text { Return to op room for clot evacu- } \\
\text { ation, decompression, or EVD/ } \\
\text { VPS placement }\end{array}$ & 3 & 3 & $1.00(0.20-5.03)$ \\
\hline
\end{tabular}

gation is susceptible to selection and assignment bias. NSAIDs were administered at the discretion of a multidisciplinary care team on a case-by-case basis, not as part of an institutional postoperative pain management protocol. Similarly, the decision to return to the operating room for a hemorrhagic complication was made by the attending surgeon and is inherently susceptible to multiple situation-specific factors that guide patient management. Finally, to minimize assignment bias, we used the radiologists' interpretation at the time of image acquisition as an objective method to screen for degree of postoperative hemorrhage. The language used in imaging reports therefore limited detection of more-than-minimal hemorrhage. We addressed this limitation by directly reviewing images described with ambiguous language. However, disagreement in the interpretation of the amount of hemorrhage is not uncommon between teams, and the rate of hemorrhage not requiring intervention in this study is therefore limited by this fact. Finally, given that this study is primarily one of NSAID safety following craniotomy, there are no data here to support the notion that postoperative NSAID use decreases postoperative narcotic requirements.

\section{Conclusions}

In this retrospective cohort study of children undergoing craniotomy for tumor resection, we found no evidence that same-day NSAID use was associated with an increased risk of postoperative hemorrhage. Cohort studies and small RCTs in the literature have demonstrated no association between postoperative NSAID use and intracranial hemorrhage requiring additional surgery. Symptomatic hemorrhage following craniotomy for tumor resection is rare in children, and when used judiciously, postoperative use of NSAIDs for pain control appears to be safe in this patient population.

\section{Acknowledgments}

We thank Mr. Bruce Kall and Dr. Barry Bruss for their assistance with obtaining some of the raw data used in this manuscript.

\section{References}

1. Bennett KG, Harbaugh CM, Hu HM, et al. Persistent opioid use among children, adolescents, and young adults after common cleft operations. J Craniofac Surg. 2018;29(7):1697_ 1701.

2. Harbaugh CM, Lee JS, Hu HM, et al. Persistent opioid use among pediatric patients after surgery. Pediatrics. 2018;141(1):141.

3. Gaither JR, Leventhal JM, Ryan SA, Camenga DR. National trends in hospitalizations for opioid poisonings among children and adolescents, 1997 to 2012. JAMA Pediatr. 2016;170(12):1195-1201.

4. Cashman JN. The mechanisms of action of NSAIDs in analgesia. Drugs. 1996;52(suppl 5):13-23.

5. Dolmatova EV, Imaev AA, Lubnin AY. 'Scheduled' dosing of lornoxicam provides analgesia superior to that provided by 'on request' dosing following craniotomy. Eur J Anaesthesiol. 2009;26(8):633-637.

6. Molnár C, Simon É, Kazup Á, et al. A single preoperative dose of diclofenac reduces the intensity of acute postcraniotomy headache and decreases analgesic requirements over five postoperative days in adults: a single center, randomized, blinded trial. J Neurol Sci. 2015;353(1-2):70-73.

7. Rahimi SY, Vender JR, Macomson SD, et al. Postoperative pain management after craniotomy: evaluation and cost analysis. Neurosurgery. 2006;59(4):852-857.

8. Yadav G, Choupoo S, Das SK, et al. Evaluating the role of flupirtine for postcraniotomy pain and compare it with diclofenac sodium: a prospective, randomized, double blind, placebo-controlled study. J Neurosurg Anesthesiol. 2014;26(1):3236.

9. Bauer KA, Gerson W, Wright C IV, et al. Platelet function following administration of a novel formulation of intravenous diclofenac sodium versus active comparators: a randomized, single dose, crossover study in healthy male volunteers. J Clin Anesth. 2010;22(7):510-518.

10. Dordoni PL, Della Ventura M, Stefanelli A, et al. Effect of ketorolac, ketoprofen and nefopam on platelet function. Anaesthesia. 1994;49(12):1046-1049.

11. Niemi TT, Backman JT, Syrjälä MT, et al. Platelet dysfunction after intravenous ketorolac or propacetamol. Acta Anaesthesiol Scand. 2000;44(1):69-74.

12. Magni G, La Rosa I, Melillo G, et al. Intracranial hemorrhage requiring surgery in neurosurgical patients given ketorolac: a case-control study within a cohort (2001-2010). Anesth Analg. 2013;116(2):443-447.

13. Tanskanen P, Kyttä J, Randell T. Patient-controlled analgesia with oxycodone in the treatment of postcraniotomy pain. Acta Anaesthesiol Scand. 1999;43(1):42-45.

14. Jian M, Li X, Wang A, et al. Flurbiprofen and hypertension but not hydroxyethyl starch are associated with post-craniotomy intracranial haematoma requiring surgery. Br J Anaesth. 2014;113(5):832-839.

15. Palmer JD, Sparrow OC, Iannotti F. Postoperative hematoma: a 5-year survey and identification of avoidable risk factors. Neurosurgery. 1994;35(6):1061-1065.

16. Bauer DF, Waters AM, Tubbs RS, et al. Safety and utility of scheduled nonnarcotic analgesic medications in children undergoing craniotomy for brain tumor. Neurosurgery. 2010;67(2):353-356.

17. Richardson MD, Palmeri NO, Williams SA, et al. Routine perioperative ketorolac administration is not associated with hemorrhage in pediatric neurosurgery patients. J Neurosurg Pediatr. 2016;17(1):107-115.

18. Taylor WA, Thomas NW, Wellings JA, Bell BA. Timing of postoperative intracranial hematoma development and implications for the best use of neurosurgical intensive care. $J$ Neurosurg. 1995;82(1):48-50.

19. Dasenbrock HH, Yan SC, Chavakula V, et al. Unplanned reoperation after craniotomy for tumor: a National Surgical Quality Improvement Program analysis. Neurosurgery. 2017;81(5):761-771. 
20. Desai VR, Grossman R, Sparrow H. Incidence of intracranial hemorrhage after a cranial operation. Cureus. 2016;8(5):e616.

21. Smyth MD, Banks JT, Tubbs RS, et al. Efficacy of scheduled nonnarcotic analgesic medications in children after suboccipital craniectomy. J Neurosurg. 2004;100(2)(Suppl Pediatrics):183-186.

22. Oravec CS, Motiwala M, Reed K, et al. Big data research in neurosurgery: a critical look at this popular new study design. Neurosurgery. 2018;82(5):728-746.

23. Oravec CS, Motiwala M, Reed K, et al. In reply: Big data research in neurosurgery: a critical look at this popular new study design. Neurosurgery. 2018;82(6):E188-E189.

\section{Disclosures}

The authors report no conflict of interest concerning the materials or methods used in this study or the findings specified in this paper.

\section{Author Contributions}

Conception and design: Ahn, Nesvick, Daniels. Acquisition of data: Nesvick, Oushy. Analysis and interpretation of data: Nesvick, Oushy. Drafting the article: Nesvick. Critically revising the article: all authors. Reviewed submitted version of manuscript: all authors. Approved the final version of the manuscript on behalf of all authors: Ahn. Statistical analysis: Nesvick. Administrative/technical/material support: Nesvick, Oushy, Daniels. Study supervision: Ahn, Nesvick, Daniels.

\section{Supplemental Information}

Previous Presentations

These data were presented in part at the 2019 Pediatric Section Meeting in Scottsdale, AZ.

\section{Correspondence}

Edward S. Ahn: Mayo Clinic, Rochester, MN. ahn.edward@mayo. edu. 